\title{
Developments in Human Centered Cueing Algorithms for Control of Flight Simulator Motion Systems
}

\author{
Robert J. Telban ${ }^{*}$ and Frank M. Cardullo ${ }^{\dagger}$ \\ State University of New York \\ Binghamton, New York \\ Jacob A. Houck ${ }^{\ddagger}$ \\ NASA Langley Research Center \\ Hampton, Virginia
}

\begin{abstract}
$\underline{\text { Abstract }}$
The authors conducted further research with cueing algorithms for control of flight simulator motion systems. A variation of the so-called optimal algorithm was formulated using simulated aircraft angular velocity input as a basis. Models of the human vestibular sensation system, i.e. the semicircular canals and otoliths, are incorporated within the algorithm. Comparisons of angular velocity cueing responses showed a significant improvement over a formulation using angular acceleration input. Results also compared favorably with the coordinated adaptive washout algorithm, yielding similar results for angular velocity cues while eliminating false cues and reducing the tilt rate for longitudinal cues. These results were confirmed in piloted tests on the current motion system at NASA-Langley, the Visual Motion Simulator (VMS). Proposed future developments by the authors in cueing algorithms are revealed. The new motion system, the Cockpit Motion Facility (CMF), where the final evaluation of the cueing algorithms will be conducted, is also described.
\end{abstract}

* PhD. Candidate, Department of Mechanical

Engineering, Student Member AIAA

${ }^{\dagger}$ Associate Professor, Department of Mechanical Engineering, Associate Fellow AIAA

\$Systems Development Branch, Associate Fellow AIAA

Copyright (C) 1999 by the American Institute of Aeronautics and Astronautics, Inc. No copyright is asserted in the United States under Title 17, U.S. Code. The U.S. Government has a royalty-free license to exercise all rights under the copyright claimed herein for Governmental purposes. All other rights are reserved by the copyright owner.

\section{$\underline{\text { Introduction }}$}

While a visual system alone can provide motion cues at low frequency, physical motion stimuli are necessary to provide higher frequency cues in the range sensitive to the vestibular and somatosensory systems. The addition of high fidelity motion cues from a moving platform in conjunction with visual motion cues have been shown to produce a rapid onset of vection, or the illusion of motion, thus reducing the delay associated with visual motion alone.

A key element in providing physical stimuli in flight simulators is the cueing algorithm that produces the drive signals used to control the motion system hardware. Two viable approaches to motion cueing algorithm development have been identified from research conducted by $\mathrm{Wu}$ and Cardullo. ${ }^{1,2}$

The first technique is a modification of the coordinated adaptive washout algorithm developed by Parrish, et al., hereafter referred to as the "adaptive algorithm". 3 This algorithm uses both first and second order linear washout filters in conjunction with an optimization method that adjusts the filter gains in real time by minimizing the error between the simulated vehicle and the motion platform responses. This methodology effectively produces a set of nonlinear washout filters.

The second technique is the "optimal algorithm" based on that which was developed by Sivan, et al. ${ }^{4}$ and later implemented by Reid and Nahon. ${ }^{5}$ This algorithm uses higher order filters that are developed, prior to real time application, using optimal control methods. This method incorporates a mathematical model of the human vestibular system, constraining the sensation error between the simulated aircraft and motion platform dynamics.

In their research $\mathrm{Wu}$ and Cardullo ${ }^{1,2}$ made several modifications to the optimal algorithm implemented by Reid and Nahon ${ }^{4}$, resulting in improved performance. 
The center of rotation of the motion platform was moved from the pilot's head to the motion base centroid, reducing actuator extension lengths during simulation. In the algorithm development additional states were added to the cost function to enable more flexibility in tuning the algorithm. A nonlinear gain algorithm was developed that scales the aircraft inputs by a third-order polynomial, maximizing the motion cues while remaining within the operational limits of the motion system.

The question has arisen as to what aircraft and simulator control inputs are the most appropriate for the optimal algorithm. The previous developments ${ }^{4,5}$ centered on a control input for either the longitudinal or lateral mode with linear acceleration and angular displacement as control inputs. $\mathrm{Wu}^{2}$ developed an approach using linear acceleration and angular acceleration for the longitudinal mode. This approach shows advantages in controlling additional motion states that were not available in the original development. In addition, since the semicircular canals behave as a transducer for angular velocity input in the range of normal head movements, ${ }^{6}$ an approach using angular velocity as input may also be desired.

In this paper an optimal algorithm based on simulated aircraft angular velocity inputs is discussed. Models of the human vestibular system, i.e. the semicircular canals and otoliths, are incorporated within the algorithm in order to constrain vestibular sensation errors. A set of cueing filters is optimized and generated prior to real time implementation.

Motion platform responses generated by this revised optimal algorithm are compared with responses from the optimal algorithm based on angular acceleration input. An objective comparison of motion platform responses and pilot sensation responses (as computed from the vestibular models employing platform motion as a stimulus) was made with responses generated from the adaptive algorithm. The algorithms were then tested on the NASA Langley
Visual Motion Simulator in a series of piloted test maneuvers.

\section{$\underline{\text { Visual Motion Simulator }}$}

The Visual Motion Simulator (VMS), shown in Figure 1, is a general-purpose simulator consisting of a two-crewmember cockpit mounted on a 60-inch stroke six-degree-of-freedom synergistic motion base $\mathrm{e}^{7,8}$. Motion cues are provided in the simulator by the relative extension or retraction of the six hydraulic actuators of the motion base. Both the adaptive and optimal algorithms were used to drive the motion base during this study.

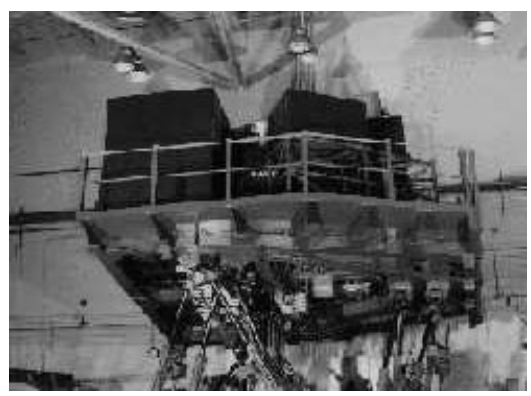

Figure 1. Visual Motion Simulator (VMS).

The cockpit of the VMS, shown in Figure 2, is designed to accommodate a generic transport aircraft configuration on the left side and a generic fighter or rotorcraft configuration on the right side. Both sides of the cockpit are outfitted with three heads-down CRT displays (primary flight display, navigation/map display, and engine display), a number of small standard electromechanical circular instruments and a control display unit mounted in the center. The left side contains a two-axis side stick control loader, and the right side contains a two-axis center stick. Both sides contain control loaded rudder systems. A center aisle stand with throttle quadrant is also available. The cockpit is outfitted with four collimated window display systems to provide an out-the-window visual scene which is driven by an Evans and Sutherland ESIG 3000/GT computer generated image system. The left side of the cockpit was used during the cueing algorithm evaluation study. 


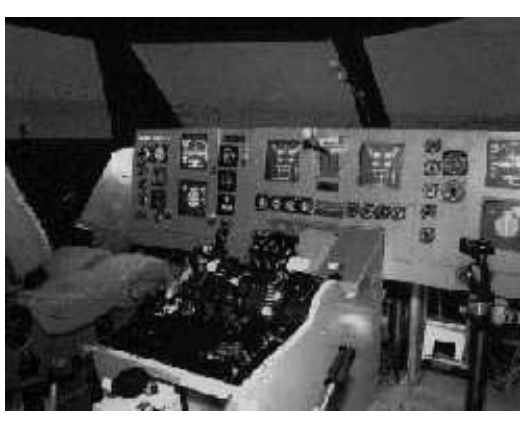

Figure 2. Visual Motion Simulator Cockpit.

The simulator includes a nonlinear mathematical model of a Boeing 737-100 aircraft, complete with landing gear dynamics, gust and wind models, radio navigation system models, and instrument and microwave landing system models. ${ }^{9}$

\section{Algorithm Development}

In developing an optimal washout filter, the problem is to determine a matrix of linear transfer functions W(s) that relates the simulator motion input to the aircraft motion input so that a cost function constraining both the sensation error between the aircraft and simulator pilot is minimized. The structure of the problem is illustrated in Figure 3.

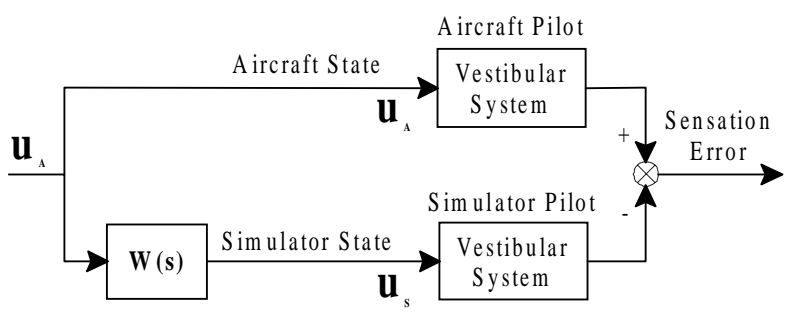

Figure 3. Aircraft Simulation Problem Structure.

A mathematical model of the human vestibular system is used in the filter development. The optimal algorithm described below generates the optimized transfer functions $\mathbf{W}(\mathbf{s})$ by an off-line MATLAB ${ }^{\mathrm{TM}}$ and

\footnotetext{
${ }^{\text {TM }}$ MATLAB and SIMULINK are registered trademarks of the Mathworks, Inc., 24 Prime Park Way, Natick, MA 01760.
}

SIMULINK $^{\mathrm{TM}}$ setup. $\mathbf{W}(\mathbf{s})$ is then implemented on-line. $\mathbf{W}(\mathbf{s})$ will relate the simulator motion input to the aircraft motion input by $\mathbf{u}_{\mathrm{s}}=\mathbf{W}(\mathbf{s}) \times \mathbf{u}_{\mathrm{a}}$. The simulator states $\mathbf{u}_{\mathbf{s}}$ are then used to generate the desired motion base commands.

The filters for four modes: longitudinal (pitch/surge), lateral (roll/sway), yaw, and heave are designed separately in the optimal algorithm development. The algorithm development with angular velocity input for the longitudinal mode is given below. The control input $\mathbf{u}$ is formulated as

$$
\mathbf{u}=\left[\begin{array}{c}
\dot{\theta} \\
a_{x}
\end{array}\right]=\left[\begin{array}{l}
u_{1} \\
u_{2}
\end{array}\right]
$$

The sensed rotational motion (pitch) is then related to the input $u_{1}$ by the semicircular canals model ${ }^{5,10}$

$$
\hat{q}=\frac{G_{s} \tau_{a} s^{2}\left(1+\tau_{L} s\right)}{\left(1+\tau_{a} s\right)\left(1+\tau_{1} s\right)\left(1+\tau_{2} s\right)} u_{1}
$$

Note that the short time constant $\tau_{2}$, equal to 0.005 seconds, ${ }^{11}$ must be included in the model, otherwise the system equation becomes non-realizable. $\tau_{2}$ was neglected by $\mathrm{Wu}^{2}$ in the optimal algorithm formulation based on angular acceleration input. Eqn. (2) can be rewritten as

$$
\hat{q}=\frac{T_{3} s^{3}+T_{4} s^{2}}{s^{3}+T_{2} s^{2}+T_{1} s+T_{0}} u_{1}
$$

where

$$
\begin{aligned}
& T_{0}=\frac{1}{\tau_{a} \tau_{1} \tau_{2}}, \quad T_{1}=\frac{\tau_{a}+\tau_{1}+\tau_{2}}{\tau_{a} \tau_{1} \tau_{2}}, \\
& T_{2}=\frac{\tau_{1} \tau_{2}+\tau_{a}\left(\tau_{1}+\tau_{2}\right)}{\tau_{a} \tau_{1} \tau_{2}} \\
& T_{3}=G_{S} \tau_{a} \tau_{L} T_{0}, \quad T_{4}=G_{S} \tau_{a} T_{0}
\end{aligned}
$$


and can be defined in state space notation as

$$
\begin{aligned}
& \mathbf{x}_{1 \sim 3}=\left[\begin{array}{lll}
x_{1} & x_{2} & x_{3}
\end{array}\right]^{\mathrm{T}} \\
& \dot{\mathbf{x}}_{1 \sim 3}=\mathbf{A}_{\operatorname{scc}} \mathbf{x}_{1 \sim 3}+\mathbf{B}_{\text {scc }} \mathbf{u} \\
& \hat{q}=\mathbf{C}_{\mathbf{s c c}} \mathbf{x}_{1 \sim 3}+\mathbf{D}_{\text {scc }} \mathbf{u}
\end{aligned}
$$

where in canonical observer form

$$
\begin{aligned}
\mathbf{A}_{\mathrm{scc}} & =\left[\begin{array}{lll}
-T_{2} & 1 & 0 \\
-T_{1} & 0 & 1 \\
-T_{0} & 0 & 0
\end{array}\right], \quad \mathbf{B}_{\text {scc }}=\left[\begin{array}{cc}
T_{4}-T_{2} T_{3} & 0 \\
-T_{1} T_{3} & 0 \\
-T_{0} T_{3} & 0
\end{array}\right] \\
\mathbf{C}_{\mathrm{scc}} & =\left[\begin{array}{lll}
1 & 0 & 0
\end{array}\right], \quad \mathbf{D}_{\mathrm{scc}}=\left[\begin{array}{ll}
T_{3} & 0
\end{array}\right]
\end{aligned}
$$

The sensed specific force (in the longitudinal axis) is now related to the aircraft specific force $f_{x}$ by the otolith model $^{5,11,12}$

$$
\hat{f_{x}}=G_{o 2} \frac{\left(s+A_{o}\right)}{\left(s+B_{o}\right)\left(s+B_{1}\right)} f_{x}
$$

For the center of rotation at the centroid of the motion platform, the specific force is

$$
f_{x}=a_{x}+g \theta+R_{S z} \ddot{\theta}
$$

where $R_{S z}$ is the radius from the motion platform centroid to the pilot's head. In terms of the control inputs $\mathrm{u}_{1}$ and $\mathrm{u}_{2}$, Eqn. (6) can be transformed into the Laplace domain

$$
f_{x}(s)=u_{2}(s)+\left(g \frac{1}{s}-R_{S_{z}} s\right) u_{1}(s)
$$

Substituting Eqn. (7) into Eqn. (5) and rearranging results in

$$
\begin{aligned}
& \hat{f}_{x}=G_{o 2} \times \\
& {\left[\frac{-R_{S z} s^{3}-R_{S_{z}} A_{0} s^{2}+g s+g A_{0}}{s\left(s+B_{0}\right)\left(s+B_{1}\right)} \frac{s+A_{0}}{\left(s+B_{0}\right)\left(s+B_{1}\right)}\right] \mathbf{u}}
\end{aligned}
$$

Note that in Eqn. (8) the system equation becomes realizable with the inclusion of the otolith break frequency $B_{1}$, which was neglected by both Reid and $\mathrm{Nahon}^{4}$ and $\mathrm{Wu}^{2}$ in their respective optimal algorithm formulations. Rearranging Eqn. (8) and taking derivatives on both sides results in the differential equation

$$
\begin{aligned}
& \ddot{\hat{f}}_{x}+\left(B_{0}+B_{1}\right) \dot{\hat{f}}_{x}+B_{0} B_{1} \hat{f}_{x}= \\
& G_{o 2} \times\left\{R_{S z}\left(B_{0}+B_{1}-A_{0}\right) \dot{u}_{1}+\left(g+R_{S z} B_{0} B_{1}\right) u_{1}\right. \\
& \left.\quad+g A_{0} \int u_{1} d t+\dot{u}_{2}+A_{0} u_{2}\right\}
\end{aligned}
$$

which can be rewritten as

$$
\begin{aligned}
& \ddot{\hat{f}}_{x}+a \dot{\hat{f}}_{x}+b \hat{f}_{x}= \\
& c \dot{u}_{1}+d u_{1}+e \int u_{1} d t+f \dot{u}_{2}+g u_{2}
\end{aligned}
$$

and can then be defined in state space notation as

$$
\begin{aligned}
& \mathbf{x}_{4 \sim 8}=\left[\begin{array}{lllll}
x_{4} & x_{5} & x_{6} & x_{7} & x_{8}
\end{array}\right]^{\mathrm{T}} \\
& \dot{\mathbf{x}}_{4 \sim 8}=\mathbf{A}_{\text {oto }} \mathbf{x}_{4 \sim 8}+\mathbf{B}_{\text {oto }} \mathbf{u} \\
& \hat{f}=\mathbf{C}_{\text {oto }} \mathbf{x}_{4 \sim 8}+\mathbf{D}_{\text {oto }} \mathbf{u}
\end{aligned}
$$

where

$$
\begin{aligned}
\mathbf{A}_{\text {oto }} & =\left[\begin{array}{ccccc}
0 & 1 & 0 & 0 & 0 \\
-b & -a & 1 & 0 & 0 \\
0 & 0 & 0 & 0 & 0 \\
0 & 0 & 0 & 0 & 1 \\
0 & 0 & 0 & -b & -a
\end{array}\right], \\
\mathbf{B}_{\text {oto }} & =\left[\begin{array}{cc}
c & 0 \\
d-a c & 0 \\
e & 0 \\
0 & f \\
0 & h-a f
\end{array}\right], \\
\mathbf{C}_{\text {oto }} & =\left[\begin{array}{llll}
1 & 0 & 1 & 0
\end{array}\right], \\
\mathbf{D}_{\text {oto }} & =G_{O 2}\left[\begin{array}{ll}
-R_{S z} & 0
\end{array}\right]
\end{aligned}
$$


The derivatives of the control input $\mathbf{u}$ are absorbed into the state space representation in Eqn. (11) by a method given in Brogan. ${ }^{13}$ The state space representations in Eqns. (4) and (11) are then combined to form a single representation for the human motion sensation model:

$$
\begin{aligned}
& \dot{\mathbf{x}}_{1 \sim 8}=\mathrm{A}_{\mathrm{v}} \mathbf{x}_{1 \sim 8}+\mathrm{B}_{\mathrm{v}} \mathrm{u} \\
& \hat{\mathbf{y}}_{1}=\mathrm{C}_{\mathrm{v}} \mathbf{x}_{1 \sim 8}+\mathrm{D}_{\mathrm{v}} \mathrm{u}
\end{aligned}
$$

where

$$
\begin{aligned}
& A_{v}=\left[\begin{array}{cc}
A_{\text {scc }} & 0 \\
0 & A_{\text {oto }}
\end{array}\right], B_{v}=\left[\begin{array}{l}
B_{s c c} \\
B_{\text {oto }}
\end{array}\right], \\
& C_{v}=\left[\begin{array}{cc}
C_{s c c} & 0 \\
0 & C_{\text {oto }}
\end{array}\right], D_{v}=\left[\begin{array}{l}
D_{\text {scc }} \\
D_{\text {oto }}
\end{array}\right]
\end{aligned}
$$

It is assumed that the same sensation model can be applied to both the pilot in the aircraft and the pilot in the simulator as shown in Figure 1. We then define the state error $\mathbf{x}_{\mathbf{e}}=\mathbf{x}_{\mathbf{s}}-\mathbf{x}_{\mathbf{a}}$ and the pilot sensation error $\mathbf{e}$, resulting in

$$
\begin{aligned}
\dot{\mathbf{x}}_{\mathrm{e}} & =\mathrm{A}_{\mathrm{v}} \mathbf{x}_{\mathrm{e}}+\mathrm{B}_{\mathrm{v}} \mathbf{u}_{\mathrm{s}}-\mathbf{B}_{\mathrm{v}} \mathbf{u}_{\mathrm{a}} \\
\mathrm{e} & =\mathrm{C}_{\mathrm{v}} \mathbf{x}_{\mathrm{e}}+\mathrm{D}_{\mathrm{v}} \mathbf{u}_{\mathrm{s}}-\mathbf{D}_{\mathrm{v}} \mathbf{u}_{\mathrm{a}}
\end{aligned}
$$

It is also necessary for the control algorithm to explicitly access motion states such as the linear velocity and displacement of the simulator, which are desired to appear in the cost function. For this purpose additional terms are included in the state equations

$$
\begin{aligned}
& \mathbf{x}_{\mathrm{d}}=\left[\begin{array}{llll}
x_{9} & x_{10} & x_{11} & x_{12}
\end{array}\right]^{\mathrm{T}} \\
& =\left[\iiint a_{x} d t^{3} \quad \iint a_{x} d t^{2} \quad \int a_{x} d t \quad \theta\right]^{\mathrm{T}} \\
& \dot{\mathbf{x}}_{\mathrm{d}}=\mathbf{A}_{\mathrm{d}} \mathbf{x}_{\mathrm{d}}+\mathbf{B}_{\mathrm{d}} \mathbf{u}
\end{aligned}
$$

where

$$
\mathbf{A}_{\mathbf{d}}=\left[\begin{array}{lllll}
0 & 1 & 0 & 0 & 0 \\
0 & 0 & 1 & 0 & 0 \\
0 & 0 & 0 & 0 & 0 \\
0 & 0 & 0 & 0 & 1 \\
0 & 0 & 0 & 0 & 0
\end{array}\right], \mathbf{B}_{d}=\left[\begin{array}{ll}
0 & 0 \\
0 & 0 \\
0 & 1 \\
0 & 0 \\
1 & 0
\end{array}\right]
$$

Input $\mathrm{u}$ consists of filtered white noise, and can be expressed in state space as

$$
\begin{gathered}
\dot{\mathbf{x}}_{\mathrm{n}}=\mathrm{A}_{\mathrm{n}} \mathbf{x}_{\mathrm{n}}+\mathbf{B}_{\mathrm{n}} \mathbf{W} \\
\mathbf{u}=\mathbf{x}_{\mathrm{n}}
\end{gathered}
$$

where

$$
\mathbf{A}_{\mathrm{n}}=\left[\begin{array}{cc}
-1.0 & 0 \\
0 & -4 \pi
\end{array}\right], \mathbf{B}_{\mathrm{n}}=\left[\begin{array}{l}
1.0 \\
1.0
\end{array}\right]
$$

The state equations given in Eqns. (13), (14), and (15) can be combined to form the desired system equation

$$
\begin{aligned}
& \mathbf{x}=\left[\begin{array}{lll}
\mathbf{x}_{\mathrm{e}} & \mathbf{x}_{\mathrm{d}} & \mathbf{x}_{\mathrm{n}}
\end{array}\right]^{\mathrm{T}} \\
& \dot{\mathbf{x}}=\mathbf{A} \mathbf{x}+\mathbf{B} \mathbf{u}_{\mathrm{s}}+\mathbf{H} \mathbf{w} \\
& \mathbf{y}=\left[\begin{array}{ll}
\mathbf{e} & \mathbf{x}_{\mathrm{d}}
\end{array}\right]^{\mathrm{T}}=\mathbf{C} \mathbf{x}+\mathbf{D} \mathbf{u}_{\mathrm{s}}
\end{aligned}
$$

where

$$
\begin{aligned}
& \mathbf{A}=\left[\begin{array}{ccc}
\mathbf{A}_{\mathrm{V}} & \mathbf{0} & -\mathbf{B}_{\mathrm{V}} \\
\mathbf{0} & \mathbf{A}_{\mathrm{d}} & \mathbf{0} \\
\mathbf{0} & \mathbf{0} & \mathbf{A}_{\mathrm{n}}
\end{array}\right], \quad \mathbf{B}=\left[\begin{array}{c}
\mathbf{B}_{\mathrm{V}} \\
\mathbf{B}_{\mathrm{d}} \\
\mathbf{0}
\end{array}\right] \\
& \mathbf{C}=\left[\begin{array}{ccc}
\mathbf{C}_{\mathrm{V}} & \mathbf{0} & -\mathbf{D}_{\mathrm{V}} \\
\mathbf{0} & \mathrm{I} & \mathbf{0}
\end{array}\right], \quad \mathrm{H}=\left[\begin{array}{c}
\mathbf{0} \\
\mathbf{0} \\
\mathbf{B}_{\mathrm{n}}
\end{array}\right] \\
& \mathbf{D}=\left[\begin{array}{ll}
\mathbf{D}_{\mathrm{V}} & 0
\end{array}\right]
\end{aligned}
$$


with the cost function

$$
J=E\left\{\int_{t_{0}}^{t_{1}}\left(\mathbf{e}^{\mathrm{T}} \mathbf{Q} \mathbf{e}+\mathbf{x}_{\mathrm{d}}^{\mathrm{T}} \mathbf{R}_{\mathrm{d}} \mathbf{x}_{\mathrm{d}}+\mathbf{u}_{\mathrm{s}}^{\mathrm{T}} \mathbf{R} \mathbf{u}_{\mathrm{s}}\right) d t\right\}
$$

where $E$ is the average or expected value. Eqn. (17) implies that three variables are to be constrained in the cost function: the sensation error $\mathbf{e}$ along with the additional terms $\mathbf{x}_{\mathbf{d}}$ and $\mathbf{u}_{\mathbf{s}}$ which together define the linear and angular motion of the platform.

The system equation of Eqn. (16) and the cost function of Eqn. (17) can be transformed to the standard optimal control form ${ }^{14}$ by the following equations:

$$
\begin{aligned}
& \dot{\mathbf{x}}=\mathbf{A}^{\prime} \mathbf{x}+\mathbf{B} \mathbf{u}^{\prime}+\mathbf{H} \mathbf{w} \\
& J=E\left\{\int_{t_{0}}^{t_{1}}\left(\mathbf{x}^{\mathrm{T}} \mathbf{R}_{1}^{\prime} \mathbf{x}+\mathbf{u}^{\prime \mathrm{T}} \mathbf{R}_{2} \mathbf{u}^{\prime}\right) d t\right\}
\end{aligned}
$$

where

$$
\begin{aligned}
& \mathbf{G}=\left[\begin{array}{cc}
\mathbf{Q} & \mathbf{0} \\
\mathbf{0} & \mathbf{R}_{\mathrm{d}}
\end{array}\right], \\
& \mathbf{R}_{1}=\mathbf{C}^{\mathrm{T}} \mathbf{G} \mathbf{C}, \mathbf{R}_{2}=\mathbf{R}+\mathbf{D}^{\mathrm{T}} \mathbf{G} \mathbf{D}, \\
& \mathbf{A}^{\prime}=\mathbf{A}-\mathbf{B} \mathbf{R}_{2}^{-1} \mathbf{R}_{12}^{\mathrm{T}}, \mathbf{u}^{\prime}=\mathbf{u}_{\mathrm{s}}+\mathbf{R}_{2}^{-1} \mathbf{R}_{12}^{\mathrm{T}} \mathbf{x}, \\
& \mathbf{R}_{1}^{\prime}=\mathbf{R}_{1}-\mathbf{R}_{12} \mathbf{R}_{2}^{-1} \mathbf{R}_{12}^{\mathrm{T}}
\end{aligned}
$$

The cost function of Eqn. (18) is minimized when

$$
\mathbf{u}^{\prime}=-\mathbf{R}_{2}^{-1} \mathbf{B}^{\mathrm{T}} \mathbf{P} \mathbf{x}
$$

and defining the optimal feedback gain matrix $\mathbf{F}$,

$$
\mathbf{F}=\mathbf{R}_{2}^{-1}\left(\mathbf{B}^{\mathrm{T}} \mathbf{P}+\mathbf{R}_{12}^{\mathrm{T}}\right)
$$

F can then be partitioned corresponding to the partition of $\mathbf{x}$ in Eqn. (16):

$$
\mathbf{u}_{\mathrm{s}}=-\left[\begin{array}{lll}
\mathbf{F}_{1} & \mathbf{F}_{2} & \mathbf{F}_{3}
\end{array}\right]\left[\begin{array}{l}
\mathbf{x}_{\mathrm{e}} \\
\mathbf{x}_{\mathrm{d}} \\
\mathbf{x}_{\mathrm{n}}
\end{array}\right]+\left[\begin{array}{l}
\mathbf{B}_{\mathrm{v}} \\
\mathbf{B}_{\mathrm{d}}
\end{array}\right] \mathbf{u}_{\mathrm{s}}
$$

Remove the states corresponding to the $\mathbf{x}_{\mathbf{n}}$ partition from Eqn. (16):

$$
\left[\begin{array}{c}
\dot{\mathbf{x}}_{\mathrm{e}} \\
\dot{\mathbf{x}}_{\mathrm{d}}
\end{array}\right]=\left[\begin{array}{ccc}
\mathbf{A}_{\mathrm{v}} & \mathbf{0} & -\mathbf{B}_{\mathrm{v}} \\
\mathbf{0} & \mathbf{A}_{\mathrm{d}} & \mathbf{0}
\end{array}\right]\left[\begin{array}{l}
\mathbf{x}_{\mathrm{e}} \\
\mathbf{x}_{\mathrm{d}} \\
\mathbf{x}_{\mathrm{n}}
\end{array}\right]+\left[\begin{array}{l}
\mathbf{B}_{\mathrm{v}} \\
\mathbf{B}_{\mathrm{d}}
\end{array}\right] \mathbf{u}_{\mathrm{s}}
$$

After taking the Laplace transform of Eqns. (23) and (24), the following equations are obtained:

$$
\mathbf{u}_{\mathrm{a}}(\mathbf{s})=\mathrm{W}(\mathbf{s}) \mathbf{u}_{\mathrm{s}}(\mathbf{s})
$$

where

$$
\begin{aligned}
& W(s)=\left[\begin{array}{ll}
F_{1} & F_{2}
\end{array}\right] \times \\
& {\left[\begin{array}{cc}
s I-A_{v}+B_{v} F_{1} & B_{v} F_{2} \\
B_{d} F_{1} & s I-A_{d}+B_{d} F_{2}
\end{array}\right]^{-1} \times} \\
& {\left[\begin{array}{c}
B_{v}\left(I+F_{3}\right) \\
B_{d} F_{3}
\end{array}\right]-F_{3}}
\end{aligned}
$$

The optimal filter matrix $\mathbf{W}(\mathbf{s})$ is computed using a set of MATLAB scripts. The weighting matrices $\mathbf{Q}, \mathbf{R}$, and $\mathbf{R}_{\mathbf{d}}$ given in the cost function of Eqn. (17) are selected and adjusted to produce the desired platform responses. From these weights and the vestibular models the standard optimal control matrices of Eqn. (18) are computed. The algebraic Riccati equation of Eqn. (19) is solved with the MATLAB function "care". The solution for $\mathbf{W}(\mathbf{s})$ in Eqn. (25) is then computed. Common poles and zeroes are cancelled in each transfer function, yielding a set of seventh-order filters for the longitudinal mode. These filters are then used in a 
SIMULINK model that generates the linear acceleration and angular velocity responses.

\section{Algorithm Evaluation}

Comparisons of longitudinal responses are made between the optimal algorithm with angular velocity input and the optimal algorithm with angular acceleration input proposed by $\mathrm{Wu}^{2}$. Both algorithms incorporate the vestibular models described in the previous section and employ identical polynomial coefficients for scaling the aircraft input. The cost function weights are kept the same for both algorithms and tuned to produce optimum responses for both pitch and surge inputs. The translational break frequency as given in Eqn. (15) was increased from $1 \mathrm{rad} / \mathrm{s}$ to $4 \pi$ $\mathrm{rad} / \mathrm{s}$ in both algorithms to minimize an onset false cue for responses to a surge step input.

Figure 4 compares specific force responses in the $\mathrm{x}$-axis (positive sense forward) for an aircraft surge ramp to step input of $1 \mathrm{~m} / \mathrm{s}^{2}$ magnitude and $3 \mathrm{~m} / \mathrm{s}^{2} / \mathrm{s}$ slope. Note that the responses are nearly identical with no onset false cue. Figure 5 compares responses to a pitch acceleration doublet input of $0.05 \mathrm{rad} / \mathrm{s}^{2}$ magnitude for a 5-second duration. Note that for the angular acceleration algorithm the pulse doublet is directly input to the rotational filter $\mathrm{W}_{11}$, producing a discontinuity at the doublet transition points. For the angular acceleration the pulse doublet is first integrated to a smooth triangular angular velocity which is then input to the filter $\mathrm{W}_{11}$.

Objective comparison of responses to calibrated aircraft inputs for the optimal algorithm with angular velocity input are made with the adaptive algorithm. For both algorithms a time step of 0.025 seconds (an update rate of $40 \mathrm{~Hz}$ ) was chosen to match the NASA Boeing 737-100 simulation. Polynomial scaling coefficients for each algorithm are tuned separately to optimize performance for the actuator stroke limits of the VMS. Comparisons are made of both specific force at the pilot's head and platform angular velocity, as well as vestibular responses.

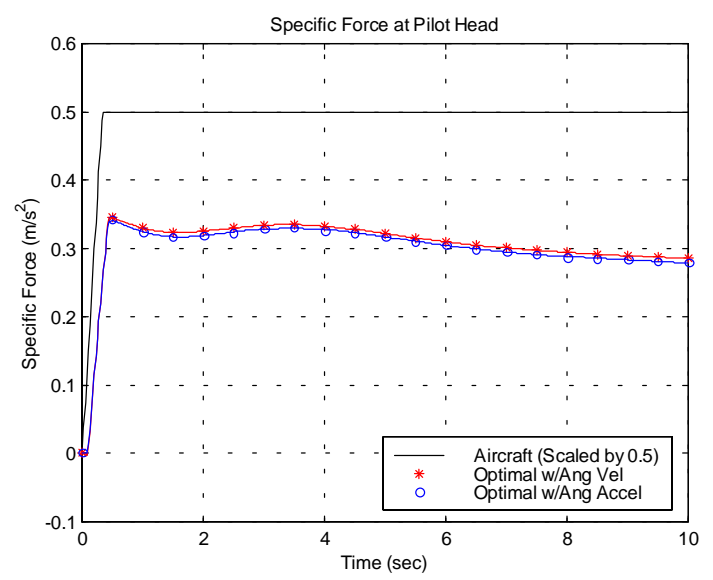

Figure 4. Comparison of Optimal Algorithm Responses to Aircraft Surge Input.

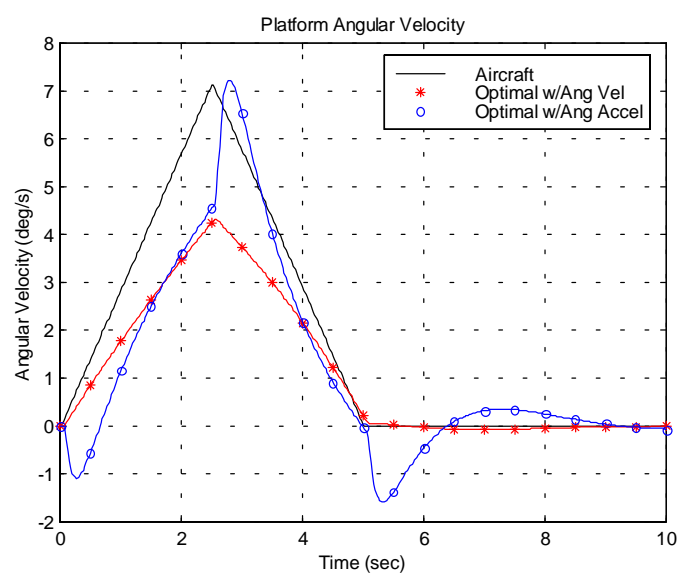

Figure 5. Comparison of Optimal Algorithm Responses to Aircraft Pitch Input.

The specific force responses to a ramp to step surge input of magnitude $1 \mathrm{~m} / \mathrm{s}^{2}$ and slope $3 \mathrm{~m} / \mathrm{s}^{2} / \mathrm{s}$ are shown in Figure 6. The adaptive algorithm produces a significant false cue $\left(-0.5 \mathrm{~m} / \mathrm{s}^{2}\right)$ at onset, after which the peak is followed by a "sag" (decrease followed by increase) for about 5 seconds until a steady magnitude is reached. The optimal algorithm produces no false cue with a smooth ramp at onset followed by a smaller peak magnitude and faster washout. The sensed specific force responses show the simulator pilot response from the optimal algorithm, while reduced in magnitude, closely tracks the shape of the perceived response of the aircraft pilot. The adaptive algorithm 
does not track the shape of the aircraft pilot sensed response as well, especially for the duration where the sag occurred.
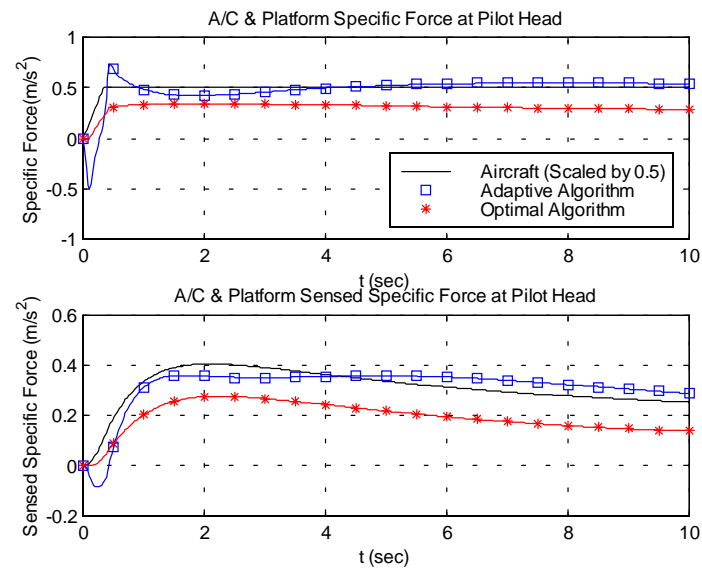

Figure 6. Comparison of Adaptive and Optimal Algorithm Responses to Aircraft Surge Input.

Angular velocity (pitch) responses due to tilt coordination generated by the surge cue are shown in Figure 7. The responses show a lower peak velocity at onset for the optimal algorithm by about $1 \mathrm{deg} / \mathrm{s}$ but followed by a negative peak of about $1 \mathrm{deg} / \mathrm{s}$ before the platform settles to zero velocity. The adaptive algorithm settles to zero velocity with no negative peak.
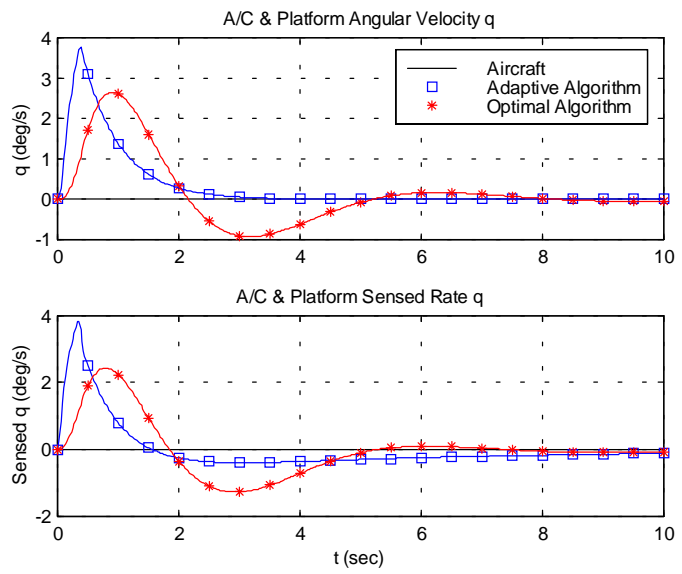

Figure 7. Platform Tilt Coordination Responses to Aircraft Surge Input.
Figure 8 shows the angular velocity responses to a pitch acceleration doublet input of $0.05 \mathrm{rad} / \mathrm{s}^{2}$ magnitude for a 5-second duration. The algorithm responses are nearly identical; each response is a proportionately reduced magnitude of the aircraft angular velocity input. Figure 9 shows the specific force response in the $\mathrm{z}$-axis (positive sense down) due to the pitch cue. Note that the response for the optimal algorithm is smaller in magnitude (and closer to the aircraft response) as compared to the adaptive algorithm response; this is consistent with the slightly larger pitch cue shown in Figure 7.
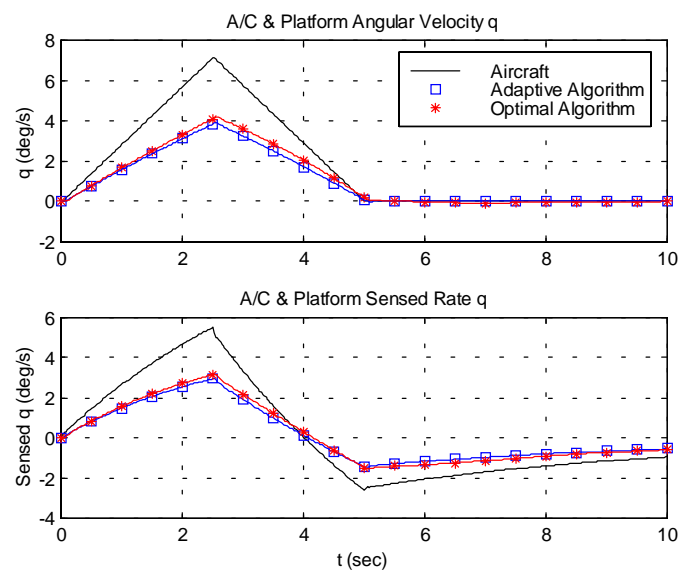

Figure 8. Comparison of Adaptive and Optimal Algorithm Responses to Aircraft Pitch Input.
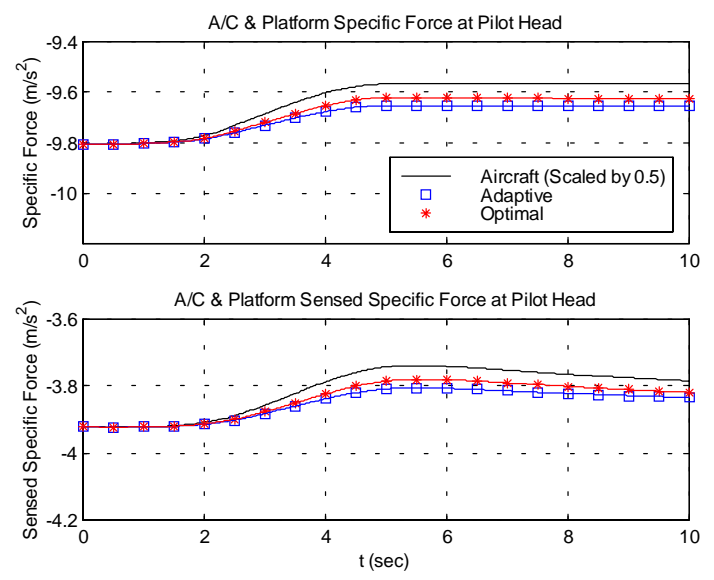

Figure 9. Z-axis Specific Force Responses to Aircraft Pitch Input. 
Both the adaptive and optimal algorithms were implemented in the real time environment on the VMS and were tested with piloted simulation maneuvers. The pilot was required to execute a series of prescribed maneuvers for each algorithm. One such maneuver is a column doublet in which the pilot uses the column control input to pitch the aircraft down and then up.

Figure 10 shows the angular velocity response for the pilot maneuver executed with the optimal algorithm. For comparison the adaptive algorithm response was generated off-line from the recorded aircraft input, producing a predicted response to compare with the measured response recorded for the optimal algorithm. Note that the adaptive algorithm has an angular velocity greater than zero at the onset that results from a tilt response generated from the aircraft trim acceleration input. On the VMS the motion platform pitch angle is adjusted to the trim inputs during a "hold" mode prior to the start of a maneuver, with the platform already at rest when the maneuver is executed in "operate" mode.
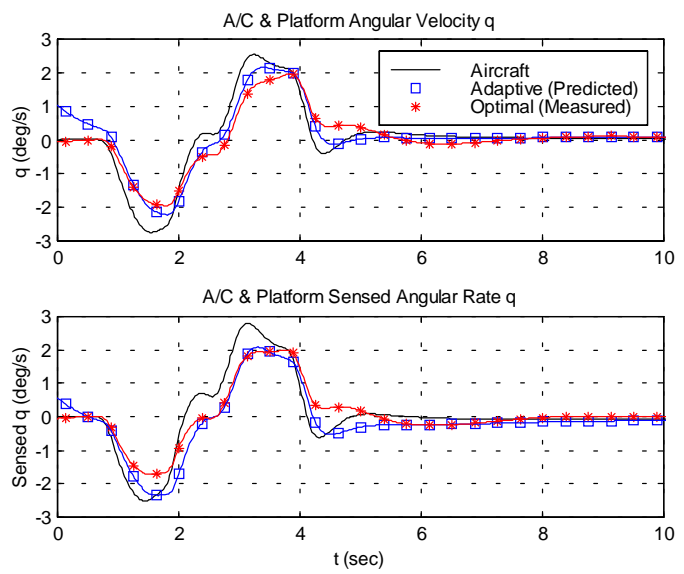

Figure 10. Algorithm Comparison of Pilot Test Column Doublet Maneuver.

As shown in Figure 10 both algorithms track the high magnitude, low frequency peaks fairly well. The adaptive algorithm does a better job at "adapting" to low magnitude, high frequency variations; especially the peak at about 2.5 seconds and the settling effect at about 4 seconds.
Figure 11 compares the specific force response in the z-axis for the column doublet maneuver. Note that the aircraft specific force starts at a value of less magnitude than the algorithm responses due to the tilt angle generated from the aircraft trim acceleration input. The aircraft input is also scaled by 10 per cent since the cueing algorithms produce a much smaller heave response due to motion platform excursion limits. Both algorithms perform about the same in tracking the large heave input peak from the aircraft, with the response washing out very quickly.
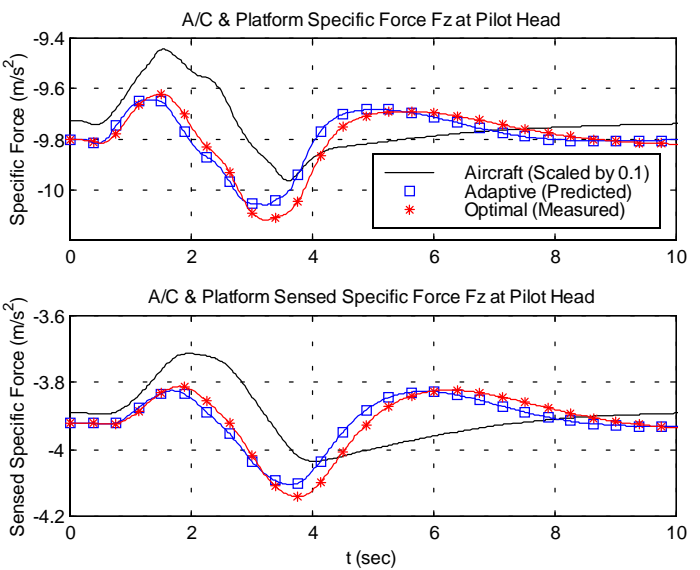

Figure 11. Z-axis Specific Force Responses to Pilot Test Column Doublet Maneuver.

\section{Future Developments}

A novel approach to motion cueing, currently being researched by the authors, is to develop a motion cueing algorithm that combines features of both the adaptive and optimal algorithms. The algorithm would be formulated as an optimal control problem with a nonlinear control law that would result in a set of adaptive cueing filters. These cueing filters can then be adjusted in real time based upon the system states; in particular those associated with perceptual errors. The control law will require the matrix Riccati equation to be solved in real time. A highly favorable approach to this computationally challenging problem is a recurrent neural network proposed by Wang and $\mathrm{Wu}^{15}$. The proposed algorithm will also incorporate a new otoliths model and a model of visual motion perception. 
The current otoliths model given in Eqn. (5) is based upon subjective responses of test subjects. From physiological experiments, Fernandez and Goldberg developed the following transfer function for the otoliths: ${ }^{16}$

$$
\frac{A F R(s)}{f(s)}=K\left(\frac{1+k_{a} \tau_{a} s}{1+\tau_{a} s}\right)\left(\frac{1+k_{v}\left(\tau_{v} s\right)^{k_{v}}}{1+\tau_{m} s}\right)
$$

where $A F R$ is the afferent firing rate of the vestibular neuron. Note that the numerator in Eqn. (26) contains a fractional derivative term that poses an interesting problem when implementing in state space notation in the optimal algorithm.

Young $^{17}$ noted that visual motion cues are dominant in the perception of velocity and steady state orientation at low frequencies below $0.1 \mathrm{~Hz}$. At higher frequencies, vestibular cues tend to dominate. When visual and vestibular cues conflict, in particular with the direction of motion, vestibular cues will initially dominate. Motion perception can be sustained by visual cues after vestibular cues have been washed out due to motion platform limits. Visual cues introduce a bias to the perceived angular velocity in the presence of platform motion. Zacharias ${ }^{18}$ developed functional models of how visual and vestibular cues operate in conjunction to produce human motion perception.

The current adaptive and optimal algorithms along with the new algorithm will be implemented and evaluated on a new motion system in the Cockpit Motion Facility (CMF). The CMF, as shown in Figure 12 , is made up of one motion system site and four fixed-base sites. The motion system site contains a sixdegree-of-freedom state-of-the-art synergistic motion base with 76-inch extension actuators. The four fixedbase sites provide homes for the simulator cockpits when they are not resident on the motion system. Each cockpit has its own visual display system and all cockpits share Evans and Sutherland ESIG 3000 or Harmony image generators.

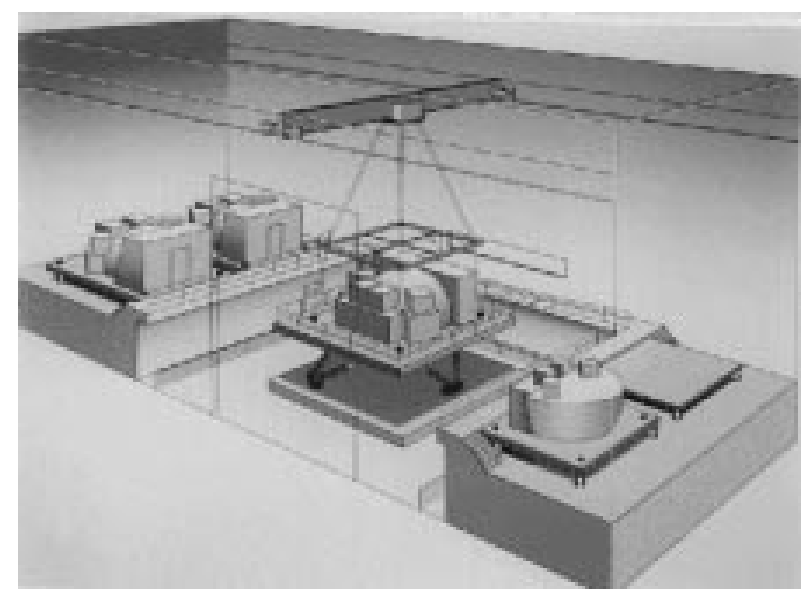

Figure 12. Cockpit Motion Facility (CMF).

The effectiveness of the proposed algorithm as compared to the current adaptive and optimal algorithms will be assessed in piloted simulations on the CMF. A series of aircraft maneuvers will be executed for each algorithm. Pilot perception (as computed from vestibular and visual motion models employing platform motion as a stimulus) and pilot control input will be recorded for each maneuver. From pilot control inputs, power spectral density, crossover frequency, and phase angle will be analyzed to determine the effect of motion platform response upon pilot performance. From these data, the fidelity of each algorithm will be benchmarked in replicating pilot performance and workload of actual aircraft maneuvers.

\section{$\underline{\text { Conclusions }}$}

Further investigation of the optimal algorithm revealed that a revised development based upon angular velocity input is an improvement over the former approach based upon angular acceleration input. Comparisons of the angular velocity response show that distortion is eliminated with the angular velocity approach, with a cueing response very close to that of the adaptive algorithm. Pilot testing on the VMS platform confirmed these results and revealed that the adaptive algorithm is more capable of tracking small changes in aircraft angular velocity inputs. Cueing responses to a surge input show the optimal algorithm 
has improved tracking capability without a false cue and lower tilt rate at onset, but producing a lower magnitude response than the adaptive algorithm.

This revised approach to the optimal algorithm will be used in the future development of an optimal algorithm that will be capable of adapting to platform motion and sensation errors in real time. This new technique will include new features not available in the current algorithms, a revised otolith model and a visual motion perception model. The new algorithm will be tested and implemented on the CMF motion platform currently being installed at the NASA Langley Research Center. Both the new motion cueing algorithm and the new motion platform facility will be instrumental in future motion studies research.

\section{$\underline{\text { References }}$}

${ }^{1} \mathrm{Wu}, \mathrm{W}$., and Cardullo, F. M., Is There an Optimum Cueing Algorithm?, AIAA Modeling and Simulation Technologies Conference, New Orleans, LA, August 11-13, 1997, pp. 23-29.

${ }^{2} \mathrm{Wu}, \mathrm{W}$., Development of Cueing Algorithm for the Control of Simulator Motion Systems, MS Thesis, State University of New York at Binghamton, May 1997.

${ }^{3}$ Martin, D. J., Jr.; A Digital Program for Motion Washout on Langley's Six-degree-of-freedom Motion Simulator, NASA Contractor Report 145219, Contract NAS1-14600, July 1977.

${ }^{4}$ Sivan, R., Ish-shalom, J. and Huang J. K., An Optimal Control Approach to the Design of Moving Flight Simulators, IEEE Transactions on Systems, Man, and Cybernetics, vol. SMC-12, no.6, November/December 1982, pp. 818-827.

${ }^{5}$ Reid, L. D. and Nahon, M. A., Flight Simulation Motion-base Drive Algorithms: Part 1 - Developing and Testing the Equations, UTIAS Report No. 296, CN ISSN 0082-5255, December 1985.

${ }^{6}$ Young, L. R.; Perception of the Body in Space: Mechanisms, Handbook of Physiology - The Nervous System, Chapter 22, pp. 1023-1066.

${ }^{7}$ Parrish, R. V., Dieudonne, J. E., Martin, D. J., Jr., and Copeland, J. L., Compensation Based on Linearized Analysis for a Six-Degree-of-Freedom Motion Simulator, NASA TND-7349, 1973.

${ }^{8}$ Parrish, R. V., Dieudonne, J. E., and Martin, D. J., Jr., Motion Software for a Synergistic Six-Degree-ofFreedom Motion Base, NASA TND-7350, 1973.
${ }^{9}$ DeLoach, R. and Houck, J. A., Pilot Evaluation of Population-Minimal Ground Tracks in the Airport Community, AIAA Journal of Aircraft, Volume 24, Number 9, September 1987.

${ }^{10}$ Zacharias, G. L., Motion Cue Models for PilotVehicle Analysis, Defense Documentation Center, Defense Logistics Agency, Cameron Station,

Alexandria, VA, May, 1978.

${ }^{11}$ Goldberg, J. M. and Fernandez, C., Physiology of Peripheral Neurons Innervating Semicircular Canals of the Squirrel Monkey. I, II, III, Journal of

Neurophysiology, vol. 34, no. 4, 1971, pp. 635-685.

${ }^{12}$ Meiry, J. L., The Vestibular System and Human Dynamic Space Orientation, NASA CR-628, October 1966.

${ }^{13}$ Brogan, W. L., Modern Control Theory, Third Edition, Prentice-Hall, 1991, pp. 82 - 83.

${ }^{14}$ Vincent, T. L., and Grantham, W. J., Nonlinear and Optimal Control Systems, John Wiley and Sons, Inc., 1997, pp. $466-469$

${ }^{15}$ Wang, J. and Wu, G., A multilayer recurrent neural network for solving continuous-time algebraic Riccati equations, Neural Networks, no. 11, 1998, pp. 939950.

${ }^{16}$ Fernandez, C., and Goldberg, J. M., Physiology of Peripheral Neurons Innervating Otolith Organs of the Squirrel Monkey. I, II, and III, Journal of Neurophysiology, vol. 39, no. 5, 1976, pp. 971-1009

${ }^{17}$ Young, L. R., Visual Vestibular Interaction, Sixth International Symposium on Biocybernetics, Control Mechanisms in Bio- and Ecosystems, International Federation of Automatic Control, Leipzig, East Germany, September 12 - 16, 1977.

${ }^{18}$ Zacharias, G. L., Motion Sensation Dependence on Visual and Vestibular Cues, PhD. Thesis, Massachusetts Institute of Technology, September 1977. 